\title{
Highly Efficient, One Pot Synthesis and Oxidation of Hantsch 1,4-Dihydropyridines Mediated by Iodobenzene Diacetate (III) Using Conventional Heating, Ultrasonic and Microwave Irradiation
}

\author{
Khalid Hussain ${ }^{*}$, Deepak Wadhwa ${ }^{2}$ \\ ${ }^{1}$ Mewat Engineering College (WAKF), Nuh, India \\ ${ }^{2}$ Department of chemistry, Kurukshetra University, Kurukshetra, India \\ Email: ${ }^{*}$ khalidchem83@yahoo.co.in
}

Received 23 June 2014; revised 10 August 2014; accepted 26 August 2014

Copyright (C) 2014 by authors and Scientific Research Publishing Inc.

This work is licensed under the Creative Commons Attribution International License (CC BY). http://creativecommons.org/licenses/by/4.0/

\section{(c) (i) Open Access}

\begin{abstract}
A mild, general, convenient, and efficient one-pot synthesis of 4-arylpyridines (4) is described using conventional heating, ultrasound and microwave irradiation. Aryl aldehydes (2) were efficiently condensed with ethylacetoacetate (1) and ammonium acetate in acetonitrile to give dihydropyridine intermediates (4). The latter underwent a smooth Iodobenzene Diacetate (III) mediated aromatization reaction in the same pot to afford 4-arylpyridines (4) in good to excellent yields.
\end{abstract}

Keywords

Dihydropyridines, Iodobenzene Diacetate, Ultrasonic, Microwave, One-Pot, Formylpyrazoles

\section{Introduction}

Development of highly efficient synthetic methodologies for the construction of biologically important compounds is one of the challenges to medicinal and organic chemist. One of the most relevant approaches to synthetic efficiency is based on multi component reactions (MCRs) which combine three or more substrates, either simultaneously, leading to domino processes [1], or through the sequential reactions without isolating interme-

\footnotetext{
${ }^{*}$ Corresponding author.
}

How to cite this paper: Hussain, K. and Wadhwa, D. (2014) Highly Efficient, One Pot Synthesis and Oxidation of Hantsch 1,4-Dihydropyridines Mediated by lodobenzene Diacetate (III) Using Conventional Heating, Ultrasonic and Microwave Irradiation. International Journal of Organic Chemistry, 4, 174- 181. http://dx.doi.org/10.4236/ijoc.2014.43019 
diate species. MCRs offer several advantages such as atom economy, minimized waste generation, because of the reduction in the number of work-up, extraction and purification stages.

Use of ultrasonic irradiation and microwave irradiation as alternative sources of energy has proved to be one of the stepping stone towards the green syntheses as it offers advantage of enhanced reactivity, shorter reaction times and higher yields of pure products compared to the traditional heating methods [2] [3]. The efficiency and expediency of MCRs leading to heterocyclic scaffolds can be increased to several times using one pot reaction profiles, greener catalysts, ultrasonic, and microwave irradiation.

1,4-Dihydropyridines (1,4-DHPs) belong to a class of nitrogen containing heterocycles having a six-membered ring. Much attention has been devoted to explore their pharmacological activities. A considerable portion of today's efforts in dihydropyridine chemistry is expanded in synthesizing reduced form of nicotinamide adenine dinucleotide (NADH) mimics, exploring the reactions and mechanisms of these compounds, and utilizing them in a variety of synthetic reactions. Newly synthesized substituted 1,4-DHPs possess other pharmacological activities such as antitumor [4], bronchodilating [5], antidiabetic [6], neurotropic [7], antianginal [8] and P-glyco protein Inhibitors [9]. The benign environmental character and easy commercial availability makes hypervalent iodine (III) reagents increasingly important for the oxidation of organic molecules [10]-[18]. These days much work has been done to explore the oxidation ability, their electrophilic properties and to develop novel reaction using hypervalent iodine compounds [19].

In view of numerous biological properties associated with 1,4-DHP and the biological importance of the oxidation step of 1,4-DHP [20], we became interested in the synthesis of some new 1,4-DHPs and their corresponding pyridine derivatives via iodine (III) mediated oxidation. In continuation of our work on the utility of iodine (III) reagents for the synthesis of various types of heterocyclic compounds, we herein carried out the synthesis of 4-arylpyridines using IBD as a greener oxidant at room temperature, ultrasonic and microwave irradiation.

\section{Result and Discussion}

The present manuscript reports the synthesis of targeted diethyl 2,6-dimethyl-4-aryl-pyridine-3,5-dicarboxylate (4a-4m), by a one pot domino process. The main aim of this manuscript is to develop efficient synthetic methodology which requires lesser reaction time and reduces the number of steps involved in the synthesis of $\mathbf{4 a}-\mathbf{4 m}$. In order to achieve our aim, synthesis of diethyl 2,6-dimethyl-4-((1,3-diphenyl)-1H-pyrazol-4-yl) pyridine-3,5-dicarboxylate was carried out by a two-step reaction. We first synthesized diethyl 2,6-dimethyl-4-((1,3-diphenyl)$1 H$-pyrazol-4-yl) pyridine-3,5-dicarboxylate (3a). For this a pseudo four component reaction of ethyl acetoacetate (1) (2.0 mmol), 1,3-diphenyl-1H-pyrazole-4-carbaldehyde (2) $(1.0 \mathrm{mmol})$ and ammonium acetate (2.2 mmol) was carried out in ethanol and the reaction mixture was allowed to reflux for 25 - 35 min. The progress of the reaction was checked by TLC using petroleum ether: ethyl acetate (85: 15, v/v) as eluent. After completion of the reaction as evident from TLC, the reaction mixture was cooled down to room temperature and the solid separated was filtered under suction to afford 2,6-dimethyl-4-((1,3-diphenyl)-1H-pyrazol-4-yl)-1,4-di-hydropyridine-3,5-dicarboxylate (1a) as the desired product in $75 \%$ yield. The product so obtained was subjected to oxidation using IBD $(1.2 \mathrm{mmol})$ in DCM at room temperature. The reaction was completed in 5 min as evident from TLC (petroleum ether: ethyl acetate $(85: 15, \mathrm{v} / \mathrm{v})$ ). After the completion of reaction as evident from TLC, the reaction mixture was washed with aqueous $\mathrm{NaHCO}_{3}$ solution. Organic phase was then separated, dried and concentrated on water bath. Crude product, thus obtained, was purified by silica gel column chromatography (petroleum ether: ethyl acetate, 97:3, v/v) to afford diethyl 2,6-dimethyl-4-((1,3-diphenyl)-1H-pyrazol-4-yl) pyridine-3,5-dicarboxylate (4a) as a pure product in $68 \%$ yield. In order to improve the overall yield of $4 \mathbf{a}$ and to reduce the time required, this method can be further expanded to a modular one-pot synthesis, without isolation of intermediate 3a, in contrast to our previous protocols. We decided to try a domino process for the synthesis of $\mathbf{4 a}$ without the isolation of $\mathbf{3 a}$ in one pot. To achieve this, firstly a mixture of ethyl acetoacetate (1) (2.0 mmol), 1, 3-diphenyl- $1 H$-pyrazole-4-carbaldehyde (2) $(1.0 \mathrm{mmol})$ and ammonium acetate $(2.2 \mathrm{mmol})$ was taken in $50 \mathrm{~mL}$ round bottomed flask using acetonitrile as solvent. The content of the flask was heated to reflux for $20-25 \mathrm{~min}$. The progress of the reaction was monitored by TLC using petroleum ether: ethyl acetate (85: 15, v/v) as eluent. After formation of 3a as confirmed by TLC, IBD $(1.2 \mathrm{mmol})$ was then added to the same reaction mixture and the reaction mixture was refluxed again for 5 - $8 \mathrm{~min}$. After the completion of reaction as evident from TLC, the reaction mixture was cooled at room temperature and washed with aqueous $\mathrm{NaHCO}_{3}$ solution. Organic phase was then separated, dried and con- 
centrated on water bath. Crude product, thus obtained, was purified by silica gel column chromatography to afford pure diethyl 2,6-dimethyl-4-((1,3-diphenyl)-1H-pyrazol-4-yl)pyridine-3,5-dicarboxylate (4a) in 85\% yield. The generality of the optimized protocol was checked by carrying out the reactions using different 3-(aryl)-1phenyl-1H-pyrazole-4-carbaldehyde and also substituted benzaldehydes containing both electron withdrawing and electron releasing substituents yielded the corresponding diethyl 2,6-dimethyl-4-arylpyridine-3,5-dicarboxylate (4a-4m) in excellent yields. (Scheme $\mathbf{1}$, Table $\mathbf{1}$ ).

The efficiencies of this protocol prompted us to explore this protocol further using Ultrasonic and microwave irradiation to reduce the present serious energy crisis in the environment. All the reactions proceeded successfully and yielded the corresponding products in excellent yield. The results obtained using ultrasonic and microwave irradiation is summarized in Table 1.

\section{Conclusion}

We have synthesized a series of 2,6-dimethyl-4-aryl-pyridine-3,5-dicarboxylate derivatives (4a-4m) by one-pot domino process in acetonitrile and IBD as a greener oxidant using conventional heating, ultrasonic and microwave irradiation. This method thus provides a one pot, facile, rapid and efficient synthesis of compounds $\mathbf{4 a - 4 m}$ which are otherwise accessible through a two-step process.

\section{Experimental}

Structures of all the compounds were identified by their spectral data. Silica gel $60 \mathrm{~F}_{254}$ (Precoated aluminium plates) from Merck were used to monitor reaction progress. Melting points were determined on a melting point apparatus and are uncorrected. IR (KBr) spectra were recorded on buck scientific IR M-500 spectrophotometer and the values are expressed as $v_{\max } \mathrm{cm}^{-1}$. The ${ }^{1} \mathrm{H}$ NMR spectra were scanned on a Bruker (300 MHz) spectrometer in $\mathrm{CDCl}_{3}$ using tetramethylsilane as an internal standard. Mass spectral data were recorded on a Waters micromass Spectrometer running under Mass Lynex version 4.0 software and equipped with an ESI source. The chemical shift values are recorded on $\delta$ scale and the coupling constants $(\mathrm{J})$ are in Hz. Ultrasonic bath $(54 \mathrm{KHz}$, $300 \mathrm{~W}, 1 \mathrm{Lt}$, capacity) of Through clean ultrasonic Pvt. Ltd. (India) was used for reactions under ultrasonic irradiation. CEM discover microwave reactor was used for reactions under microwave irradiation. Pyrazole aldehydes (3) were synthesized according to the literature method [21].

\subsection{Preparation of (4a-4m) under Conventional Heating}

A mixture of ethylacetoacetate (1) (1.0 mmol), aryl aldehyde (2) $(1.0 \mathrm{mmol})$, and ammonium acetate (2.2 mmol) was dissolved in $5 \mathrm{~mL}$ of acetonitrile in a $50 \mathrm{~mL}$ round-bottomed flask. The reaction contents were refluxed on water bath for $20-25$ min. The progress of the reaction was monitored by TLC using petroleum ether: ethyl acetate (85: 15, v/v) as eluent. After formation of 3a as evident from TLC after $25 \mathrm{~min}$, IBD (1.2 mmol) was then added to the above reaction mixture and the reaction mixture was refluxed for another 5 - 8 min. After consumption of $\mathbf{3 a}$ as evident from TLC, the reaction mixture was cooled to room temperature and washed with aqueous $\mathrm{NaHCO}_{3}$ solution. Organic phase was then separated, dried and concentrated on water bath. Crude product, thus obtained, was purified by silica gel column chromatography to afford pure diethyl 2,6-dimethyl4-aryl-pyridine-3, 5-dicarboxylate (4a-4m). All the compounds $4 \mathbf{a}-\mathbf{4 m}$ are characterized by ${ }^{1} \mathrm{H} N \mathrm{NR},{ }^{13} \mathrm{C} \mathrm{NMR}$, IR and mass data.

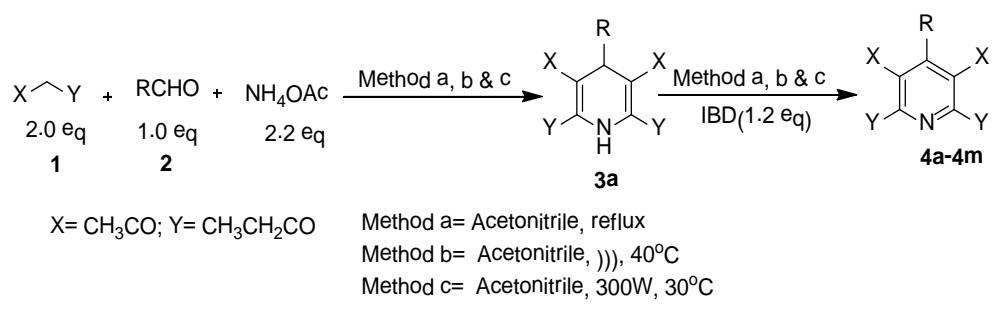

Scheme 1. Synthesis of diethyl 2,6-dimethyl-4-aryl-pyridine-3,5-dicarboxylate $(4 a-4 m)$ using one pot domino protocol. 
Table 1. Synthesis of diethyl 2,6-dimethyl-4-aryl-pyridine-3,5-dicarboxylate (4a-4m) using conventional heating, ultrasonic and microwave irradiation.

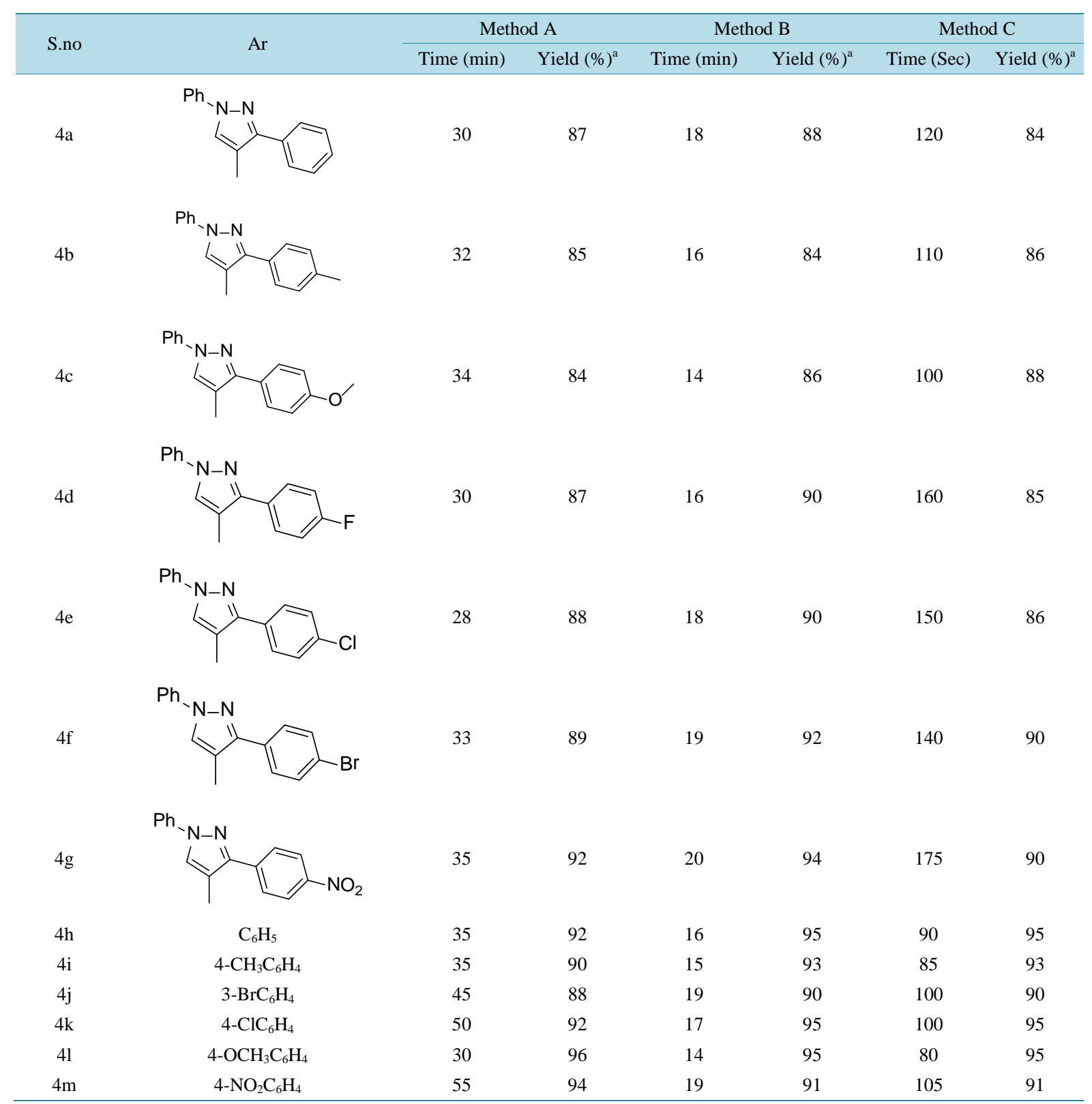

${ }^{\mathrm{a}}$ Isolated yield.

\subsection{Preparation of (4a-4m) under Ultrasonic Irradiation}

A mixture of ethylacetoacetate (1) $(1.0 \mathrm{mmol})$, aryl aldehyde (2) $(1.0 \mathrm{mmol})$, and ammonium acetate (2.2 mmol) was dissolved in $5 \mathrm{~mL}$ of acetonitrile in a $50 \mathrm{~mL}$ round-bottomed flask. The reaction contents were sonicated at $40^{\circ} \mathrm{C}$ for appropriate time as mentioned in Table 1 . The progress of the reaction was monitored by TLC using petroleum ether: ethyl acetate (85: 15, v/v) as eluent. After formation of 3a as evident from TLC after 8 min, IBD $(1.2 \mathrm{mmol})$ was then added to the above reaction mixture and the reaction mixture sonicated at room temperature for another 3 - 4 min. After consumption of $\mathbf{3 a}$ as evident from TLC the reaction mixture was washed with aqueous $\mathrm{NaHCO}_{3}$ solution. Organic phase was then separated, dried and concentrated on water bath. Crude product, thus obtained, was purified by silica gel column chromatography to afford pure diethyl 2,6-dimethyl-4-arylpyridine-3,5-dicarboxylate (4a-4m). All the compounds $4 a-4 m$ are characterized by ${ }^{1} \mathrm{H}$ NMR, ${ }^{13} \mathrm{C} N M R$, IR and 
mass data.

\subsection{Preparation of (4a-4m) under Microwave Irradiation}

A mixture of ethylacetoacetate (1) (1.0 mmol), aldehyde (2) (1.0 mmol), and ammonium acetate (2.2 mmol) was dissolved in $5 \mathrm{~mL}$ of acetonitrile in a sealed vial and placed in a CEM Discover microwave reactor. The vial was subjected to microwave irradiation, programmed at $30^{\circ} \mathrm{C}$ and $300 \mathrm{~W}$. After formation of $3 \mathbf{a}$ as evident from TLC after $1 \mathrm{~min}$, IBD (1.2 mmol) was then added to the above reaction mixture and the reaction mixture irradiated for another $30 \mathrm{sec}$. After completion of the reaction as evident from TLC, the reaction mixture was washed with aqueous $\mathrm{NaHCO}_{3}$ solution. Organic phase was then separated, dried and concentrated on water bath. Crude product, thus obtained, was purified by silica gel column chromatography to afford pure diethyl 2,6-dimethyl-4-arylpyridine-3,5-dicarboxylate (4a-4m). All the compounds $4 a-4 m$ are characterized by ${ }^{1} \mathrm{H}$ NMR, ${ }^{13} \mathrm{C} N M R$, IR and mass data.

\subsection{Characterization Data}

\subsubsection{4-(1,3-Diphenyl-1H-Pyrazol-4-yl)-2,6-Dimethyl-Pyridine-3,5-Dicarboxylic Acid Diethyl Ester (4a)}

Mp: $111^{\circ} \mathrm{C}$; IR ( $\left.v_{\max }, \mathrm{cm}^{-1}, \mathrm{KBr}\right): 1736,1233 ;{ }^{1} \mathrm{H}$ NMR (300 MHz, $\mathrm{CDCl}_{3}, \delta$, ppm): 0.95 (t, 6H, $\left.\mathrm{CH}_{3}\right), 2.613$ (s, $\left.6 \mathrm{H}, \mathrm{CH}_{3}\right), 3.910-4.07\left(\mathrm{~m}, 4 \mathrm{H}, \mathrm{OCH}_{2}\right), 7.110-7.313(\mathrm{~m}, 4 \mathrm{H}), 7.817(\mathrm{~s}, 1 \mathrm{H}), 7.581-7.690(\mathrm{~m}, 6 \mathrm{H}) ;{ }^{13} \mathrm{C}$ NMR $(75$ MHz, DMSO-d $d_{6}$ ) 166.8, 155.7, 147.8, 139.3, 138.2, 133.4, 131.2, 130.2, 129.2, 128.8, 128.0, 127.2, 118.5, 115.7, 61.7, 23.0, 13.5; Elemental analysis: Calcd for $\mathrm{C}_{28} \mathrm{H}_{27} \mathrm{~N}_{3} \mathrm{O}_{4}$ : C 71.64, H 5.76, $\mathrm{N}$ 8.95; found: C 71.63, H 5.79, N 8.93; MS (m/z): $470.20\left(\mathrm{M}^{+}+1\right)$.

\subsubsection{2,6-Dimethyl-4-(1-Phenyl-3-P-Tolyl-1H-Pyrazol-4-Yl)-Pyridine-3,5-Dicarboxylic Acid Diethyl Ester (4b)}

Mp: $105^{\circ} \mathrm{C}$; IR ( $\left.v_{\max }, \mathrm{cm}^{-1}, \mathrm{KBr}\right): 1720,1234 ;{ }^{1} \mathrm{H} \mathrm{NMR}\left(300 \mathrm{MHz}, \mathrm{CDCl}_{3}, \delta\right.$, ppm): 0.93 (t, 6H, $\left.\mathrm{CH}_{3}\right), 2.611$ (s, $\left.6 \mathrm{H}, \mathrm{CH}_{3}\right), 3.810(\mathrm{~s}, 3 \mathrm{H}), 3.99\left(\mathrm{q}, 4 \mathrm{H}, \mathrm{OCH}_{2}\right), 6.84(\mathrm{~d}, 2 \mathrm{H}, \mathrm{J}=8.7 \mathrm{~Hz}), 7.280-7.501(\mathrm{~m}, 5 \mathrm{H}), 7.732$ - $7.759(\mathrm{~d}$, $2 \mathrm{H}, \mathrm{J}=8.7 \mathrm{~Hz}$ ), 7.905 (s, $1 \mathrm{H}) ;{ }^{13} \mathrm{C}$ NMR (75 MHz, DMSO-d ${ }_{6}$ ); 167.2, 155.7, 147.9, 139.5, 137.9, 137.2, 129.8, 128.3, 127.9, 127.6, 127.3, 127.1, 119.2, 116.2, 61.2, 34.4, 23.1, 13.4; Elemental analysis: Calcd for $\mathrm{C}_{29} \mathrm{H}_{29} \mathrm{~N}_{3} \mathrm{O}_{4}$ : C 72.05, H 6.00, N 8.70; found: C 72.06, H 6.05, N 8.70; MS (m/z): $484.40\left(\mathrm{M}^{+}+1\right)$.

\subsubsection{4-[3-(4-Methoxy-Phenyl)-1-Phenyl-1H-Pyrazol-4-yl]-2,6-Dimethyl-Pyridine-3,5-Dicarboxylic Acid Diethyl Ester (4c)}

Mp: $136^{\circ} \mathrm{C}$; IR ( $\left.v_{\max }, \mathrm{cm}^{-1}, \mathrm{KBr}\right): 1740,1034 ;{ }^{1} \mathrm{H}$ NMR (300 MHz, $\mathrm{CDCl}_{3}, \delta$, ppm): 0.95 (t, 6H, $\mathrm{CH}_{3}$ ), 2.612 (s, $6 \mathrm{H}, \mathrm{CH}_{3}$ ), 3.808 (s, 3H), 4.00 (q, 4H, $\mathrm{OCH}_{2}$ ), 6.84 (d, 2H, J = 8.7 Hz), 7.311-7.501 (m, 5H), 7.74 (d, 2H, J = 8.7 $\mathrm{Hz}), 7.905$ (s, $1 \mathrm{H}) ;{ }^{13} \mathrm{C}$ NMR (75 MHz, DMSO- $\left.d_{6}\right) ; 167.1,155.9$, 148.5, 139.6, 137.8, 137.3, 129.6, 128.4, 127.9, 127.8, 127.4, 127.2, 119.0, 116.3, 61.4, 44.4, 23.0, 13.5; Elemental analysis: Calcd for $\mathrm{C}_{29} \mathrm{H}_{29} \mathrm{~N}_{3} \mathrm{O}_{5}$ : C 69.73, $\mathrm{H}$ 5.81, N 8.41; found: C 69.71, H 5.83, N 8.40; $\mathrm{MS}(\mathrm{m} / \mathrm{z}): 500.29\left(\mathrm{M}^{+}+1\right)$.

\subsubsection{4-[3-(4-Fluoro-Phenyl)-1-Phenyl-1H-Pyrazol-4-Yl]-2,6-Dimethyl-Pyridine-3,5-Dicarboxylic Acid Diethyl Ester (4d)}

Mp: $121^{\circ} \mathrm{C}$; IR (v $\left.v_{\max }, \mathrm{cm}^{-1}, \mathrm{KBr}\right): 1728,1236,1037 ;{ }^{1} \mathrm{H}$ NMR (300 MHz, $\mathrm{CDCl}_{3}, \delta$, ppm): 0.94 (t, $6 \mathrm{H}, \mathrm{CH}_{3}$ ), 2.615 (s, 6H, $\left.\mathrm{CH}_{3}\right), 3.905$ - 4.105 (q, 4H, $\mathrm{OCH}_{2}$ ), 6.987 - $7.044(\mathrm{~m}, 2 \mathrm{H}), 7.280$ - $7.365(\mathrm{~m}, 1 \mathrm{H}), 7.469$ - 7.622 (m, 4H), 7.74 (d, 2H, J = $7.8 \mathrm{~Hz}$ ), 7.923 (s, 1H); ${ }^{13} \mathrm{C}$ NMR (75 MHz, DMSO-d 6 ) 166.9, 155.6, 148.9, 139.4, 138.1, 133.3, 131.3, 130.1, 129.0, 128.9, 128.0, 127.2, 118.6, 115.8, 61.6, 23.1, 13.6; Elemental analysis: Calcd for $\mathrm{C}_{28} \mathrm{H}_{26} \mathrm{~N}_{3} \mathrm{O}_{4}$ F: C 68.99, H 5.38, N 8.62; found: C 68.95, H 5.37, N 8.63; MS (m/z): $488.36\left(\mathrm{M}^{+}+1\right)$.

\subsubsection{4-[3-(4-Chlorophenyl)-1-Phenyl-1H-Pyrazol-4-Yl]-2,6-Dimethyl-Pyridine-3,5-Dicarboxylic Acid Diethyl Ester (4e)}

Mp: $101^{\circ} \mathrm{C}-102^{\circ} \mathrm{C}\left(101^{\circ} \mathrm{C}-102^{\circ} \mathrm{C}\right.$, lit [22]); IR ( $\left.v_{\max }, \mathrm{cm}^{-1}, \mathrm{KBr}\right): 3055,2989,1747,1620,1597,1461,1322$, 1087, 1002, 952, 850, 836, 698; ${ }^{1} \mathrm{H}$ NMR (300 MHz, $\mathrm{CDCl}_{3}, \delta$, ppm): 0.935 (t, J = 7.2 Hz, 6H, $\mathrm{CH}_{3}$ ), 2.618 (s, 6H, $\left.\mathrm{CH}_{3}\right), 3.898-4.118\left(\mathrm{~m}, 4 \mathrm{H}, \mathrm{OCH}_{2}\right), 7.310-7.370(\mathrm{~m}, 2 \mathrm{H}) ; 7.487-7.513(\mathrm{~m}, 5 \mathrm{H}), 7.746(\mathrm{~d}, \mathrm{~J}=7.8 \mathrm{~Hz}, 2 \mathrm{H})$, 7.923 (s, $1 \mathrm{H}$ ); Elemental analysis: Calcd. for $\mathrm{C}_{28} \mathrm{H}_{26} \mathrm{ClN}_{3} \mathrm{O}_{4}$ : C, 66.73; H, 5.20; N, 8.34. Found: C, 61.66; H, 5.29; 
N, 8.26.

4.4.6. 4-[3-(4-Bromo-Phenyl)-1-Phenyl-1H-Pyrazol-4-Yl]-2,6-Dimethyl--Pyridine-3,5-Dicarboxylic Acid Diethyl Ester (4f)

Mp: $115^{\circ} \mathrm{C}$; IR ( $\left.v_{\max }, \mathrm{cm}^{-1}, \mathrm{KBr}\right): 1734,1030 ;{ }^{1} \mathrm{H}$ NMR (300 MHz, $\mathrm{CDCl}_{3}, \delta$, ppm): 0.95 (t, 6H, $\mathrm{CH}_{3}$ ), 2.617 (s, 6H, $\mathrm{CH}_{3}$ ), 3.99 (q, 4H, $\mathrm{OCH}_{2}$ ), 7.200 - 7.495 (m, 7H), 7.74 (d, 2H, J = $\left.7.2 \mathrm{~Hz}\right), 7.921$ (s, 1H); ${ }^{13} \mathrm{C}$ NMR $(75 \mathrm{MHz}$, DMSO-d ${ }_{6}$ ) 166.5, 155.7, 148.8, 139.6, 138.1, 132.3, 131.2, 130.0, 129.1, 128.8, 128.0, 127.1, 117.6, 115.6, 61.7, 23.4, 13.5; Elemental analysis: Calcd for $\mathrm{C}_{28} \mathrm{H}_{26} \mathrm{~N}_{3} \mathrm{O}_{4} \mathrm{Br}$ : C 61.42, H 4.75, N 7.68; found: C 61.31, H 4.79, N 7.69. MS (m/z): 548.20, 550.20.

\subsubsection{2,6-Dimethyl-4-[3-(4-Nitro-Phenyl)-1-Phenyl-1H-Pyrazol-4-Yl]-Pyridine-3,}

5-Dicarboxylicacid Diethyl Ester (4g)

Mp: $172^{\circ} \mathrm{C}$; IR ( $\left.v_{\max }, \mathrm{cm}^{-1}, \mathrm{KBr}\right): 1728,1234,1034 ;{ }^{1} \mathrm{H}$ NMR (300 MHz, $\mathrm{CDCl}_{3}, \delta$, ppm): 0.91 (t, $\left.6 \mathrm{H}, \mathrm{CH}_{3}\right)$, $2.632\left(\mathrm{~s}, 6 \mathrm{H}, \mathrm{CH}_{3}\right), 3.923-4.039\left(\mathrm{~m}, 4 \mathrm{H}, \mathrm{OCH}_{2}\right), 7.279-7.410(\mathrm{~m}, 3 \mathrm{H}), 7.499-7.769(\mathrm{~m}, 4 \mathrm{H}), 7.960(\mathrm{~s}, 1 \mathrm{H})$, 8.19 (d, $2 \mathrm{H}, \mathrm{J}=7.5 \mathrm{~Hz}) ;{ }^{13} \mathrm{C}$ NMR $\left(75 \mathrm{MHz}\right.$, DMSO-d $\left.\mathrm{d}_{6}\right) 167.9,155.8,149.0,139.8,138.2,133.6,131.5,130.3$, 129.2, 128.9, 128.0, 127.4, 119.6, 116.6, 62.5, 23.4, 13.7; Elemental analysis: Calcd for $\mathrm{C}_{28} \mathrm{H}_{26} \mathrm{~N}_{4} \mathrm{O}_{6}$ : C 64.37, $\mathrm{H}$ 4.98, N 10.73; found: C 65.34, H 5.08, N 10.87; MS (m/z): $515.26\left(\mathrm{M}^{+}+1\right)$.

\subsubsection{Diethyl-4-Phenyl-2,6-Dimethylpyridine-3,5-Dicarb Oxylate (4h)}

Mp: $62^{\circ} \mathrm{C}-63^{\circ} \mathrm{C}$; IR $\left(v_{\max }, \mathrm{cm}^{-1}, \mathrm{KBr}\right): 3026,2978,1729,1592,1477,1301,1212,1171,792,761 ;{ }^{1} \mathrm{H}$ NMR (300 $\mathrm{MHz}, \mathrm{CDCl}_{3}, \delta$, ppm): 1.22 (t, J = 7.11 Hz, 6H, $\left.\mathrm{CH}_{3}\right), 4.27$ (q, J = 7.11 Hz, 4H, OCH $)$, $2.67\left(\mathrm{~s}, 6 \mathrm{H}, \mathrm{CH}_{3}\right), 7.18$ $7.23(\mathrm{~m}, 2 \mathrm{H}), 7.30$ - 7.32 (m, 3H); Elemental analysis: Calcd. for $\mathrm{C}_{19} \mathrm{H}_{21} \mathrm{NO}_{4}$ : C, 69.71; H, 6.47; N, 4.28. Found: C, 69.88; H, 6.55; N, 4.19 .

4.4.9. Diethyl-4-(4-Methylphenyl)-2,6-Dimethylpyridine-3,5-Dicarboxylate (4i)

Mp: $70^{\circ} \mathrm{C}-71^{\circ} \mathrm{C}\left(71^{\circ} \mathrm{C}-72^{\circ} \mathrm{C}\right.$, lit [22]); IR (v $\left.v_{\max }, \mathrm{cm}^{-1}, \mathrm{KBr}\right): 3022,2978,1725,1582,1444,1228,1013,822,857$, $776 ;{ }^{1} \mathrm{H}$ NMR (300MHz, $\mathrm{CDCl}_{3}, \delta$, ppm): 1.234 (t, J = 7.11 Hz, 6H, $\left.\mathrm{CH}_{3}\right), 2.35$ (s, 3H, $\left.\mathrm{CH}_{3}\right), 2.66\left(\mathrm{~s}, 6 \mathrm{H}, \mathrm{CH}_{3}\right.$ ), 4.28 (q, J = 7.11 Hz, 4H, OCH $), 7.12(\mathrm{~d}, \mathrm{~J}=6.79 \mathrm{~Hz}, 2 \mathrm{H}), 7.23$ (d, J = 6.79 Hz, 2H); Elemental analysis: Calcd. for $\mathrm{C}_{20} \mathrm{H}_{23} \mathrm{NO}_{4}$ : C, 70.36; H, 6.79; N, 4.10. Found: C, 70.44; H, 6.84; N, 4.28.

4.4.10. Diethyl-4-(3-Bromophenyl)-2,6-Dimethylpyr-Idine-3,5-Dicarboxylate (4j)

Mp: $71^{\circ} \mathrm{C}-73^{\circ} \mathrm{C}\left(70^{\circ} \mathrm{C}-72^{\circ} \mathrm{C}\right.$, lit [22]); IR (v $\left.v_{\max }, \mathrm{cm}^{-1}, \mathrm{KBr}\right): 3055,2988,1727,1562,1280,1102,1035,866$, 777, 697; ${ }^{1} \mathrm{H}$ NMR (300MHz, $\mathrm{CDCl}_{3}, \delta$, ppm): 1.24 (t, J = 7.13 Hz, 6H, $\mathrm{CH}_{3}$ ), 4.30 (q, J = 7.13 Hz, 4H, OCH $)$, 2.66 (s, $6 \mathrm{H}, \mathrm{CH}_{3}$ ), 7.20 - 7.44 (m, 4H); Elemental analysis: Calcd. for $\mathrm{C}_{19} \mathrm{H}_{20} \mathrm{BrNO}_{4} \mathrm{C}, 56.17 ; \mathrm{H}, 4.96 ; \mathrm{N}, 3.45$. Found: C, 56.32; H, 4.88; N, 3.28.

4.4.11. Diethyl-4-(4-Chlorophenyl)-2,6-Dimethylpyr-Idine-3,5-Dicarboxylate (4k) Mp: $70^{\circ} \mathrm{C}-72^{\circ} \mathrm{C}\left(69^{\circ} \mathrm{C}-71^{\circ} \mathrm{C}\right.$, lit [22]); IR ( $\left.v_{\max }, \mathrm{cm}^{-1}, \mathrm{KBr}\right): 3028,2991,1728,1588,1232,1106,1045,857$, 657; ${ }^{1} \mathrm{H}$ NMR (300MHz, $\mathrm{CDCl}_{3}, \delta$, ppm): $1.22\left(\mathrm{t}, \mathrm{J}=7.11 \mathrm{~Hz}, 6 \mathrm{H}, \mathrm{CH}_{3}\right), 4.27$ (q, J = 7.11 Hz, 4H, $\mathrm{OCH}_{2}$ ), 2.70 (s, 6H, $\left.\mathrm{CH}_{3}\right), 7.12(\mathrm{~d}, \mathrm{~J}=8.99 \mathrm{~Hz}, 2 \mathrm{H}), 7.32(\mathrm{~d}, \mathrm{~J}=8.99 \mathrm{~Hz}, 2 \mathrm{H})$. Elemental analysis: Calcd. for $\mathrm{C}_{19} \mathrm{H}_{20} \mathrm{ClNO}_{4}: \mathrm{C}$, 63.07; H, 5.57; N, 3.87. Found: C, 62.92; H, 5.66; N, 3.66.

\subsubsection{Diethyl-4-(4-Methoxyphenyl)-2,6-Dimethyl Pyridine-3,5-Dicarboxylate (4l)}

$\mathrm{Mp}: 50^{\circ} \mathrm{C}-51^{\circ} \mathrm{C}\left(51^{\circ} \mathrm{C}-52^{\circ} \mathrm{C}\right.$, lit [22]); IR ( $\left.v_{\max }, \mathrm{cm}^{-1}, \mathrm{KBr}\right): 3034,2987,1731,1599,1523,1288,1107,856,834$, 772; ${ }^{1} \mathrm{H}$ NMR $\left(300 \mathrm{MHz}, \mathrm{CDCl}_{3}, \delta, \mathrm{ppm}\right): \mathrm{d}=1.22\left(\mathrm{t}, \mathrm{J}=7.12 \mathrm{~Hz}, 6 \mathrm{H}, \mathrm{CH}_{3}\right), 4.27$ (q, J = 7.12 Hz, 4H, OCH $)$, 2.69 (s, 6H, $\mathrm{CH}_{3}$ ), 3.86 (s, 3H, $\left.\mathrm{OCH}_{3}\right), 6.91(\mathrm{~d}, \mathrm{~J}=8.57 \mathrm{~Hz}, 2 \mathrm{H}), 7.11$ (d, J = 8.57 Hz, 2H); Elemental analysis: Calcd. for $\mathrm{C}_{20} \mathrm{H}_{23} \mathrm{NO}_{5}$ : C, 67.21; H, 6.49; N, 3.92. Found: C, 67.34; H, 6.54; N, 4.02.

\subsubsection{Diethyl-4-(4-Nitrophenyl)-2,6-Dimethylpyri-Dine-3,5-Dicarboxylate (4m)}

Mp: $110^{\circ} \mathrm{C}-112^{\circ} \mathrm{C}\left(112^{\circ} \mathrm{C}-113^{\circ} \mathrm{C}\right.$, lit [22]); IR ( $\left.v_{\max }, \mathrm{cm}^{-1}, \mathrm{KBr}\right): 3023,2988,1726,1555,1504,1351,1106$, 866, 843, 745; ${ }^{1} \mathrm{H}$ NMR $\left(300 \mathrm{MHz}, \mathrm{CDCl}_{3}, \delta, \mathrm{ppm}\right): 1.23\left(\mathrm{t}, \mathrm{J}=7.12 \mathrm{~Hz}, 6 \mathrm{H}, \mathrm{CH}_{3}\right), 2.63\left(\mathrm{~s}, 6 \mathrm{H}, \mathrm{CH}_{3}\right), 4.25(\mathrm{q}, \mathrm{J}=$ $\left.7.12 \mathrm{~Hz}, 4 \mathrm{H}, \mathrm{OCH}_{2}\right), 7.41(\mathrm{~d}, \mathrm{~J}=8.23 \mathrm{~Hz}, 2 \mathrm{H}), 8.22(\mathrm{~d}, \mathrm{~J}=8.23 \mathrm{~Hz}, 2 \mathrm{H})$. Elemental analysis: Calcd. for $\mathrm{C}_{19} \mathrm{H}_{20} \mathrm{~N}_{2} \mathrm{O}_{6}$ : C, 61.29; H, 5.41; N, 7.53. Found: C, 61.44; H, 5.32; N, 7.65. 


\section{Acknowledgements}

We are thankful to CSIR, New Delhi for the award of Junior Research Fellowship (JRF) to Khalid Hussain and KUK for Teaching Assistantship to Deepak Wadhwa.

\section{References}

[1] Tietze, L.F. (1996) Domino Reactions in Organic Synthesis. Chemical Reviews, 96, 115-136. http://dx.doi.org/10.1021/cr950027e

[2] Mason, T.J. and Meulenaer, E.C.D. (1998) Practical Considerations for Process Optimisation. Synthetic Organic Sonochemistry. Plenum Press, New York and London.

[3] Kappe, C.O. (2004) Controlled Microwave Heating in Modern Organic Synthesis. Angewandte Chemie International Edition, 43, 6250-6284. http://dx.doi.org/10.1002/anie.200400655

[4] Tsuruo, T., Iida, H., Nojiri, M., Tsukagoshi, S. and Sakurai, Y. (1983) Circumvention of Vincristine and Adriamycin Resistance in Vitro and in Vivo by Calcium Influx Blockers. Cancer Research, 43, 2905-2910.

[5] Chapman, R.W., Danko, G. and Siegels, M.I. (1984) Effect of Extra- and Intracellular Calcium Blockers on Histamine and Antigen-Induced Bronchospasms in Guinea Pigs and Rats. Pharmacology, 29, 282-291. http://dx.doi.org/10.1159/000138024

[6] Malaise, W.J. and Mathias, P.C.F. (1985) Stimulation of Insulin Release by an Organic Calcium Agonist. Diabetologia, 28, 153-156.

[7] Krauze, A., Germane, S., Eberlins, O., Sturms, I., Klusa, V. and Duburs, G. (1999) Derivatives of 3-Cyano-6-Phenyl4-(3'-Pyridyl)-Pyridine-2(1H)-Thione and Their Neurotropic Activity. European Journal of Medicinal Chemistry, 34, 301-310. http://dx.doi.org/10.1016/S0223-5234(99)80081-6

[8] Peri, R., Padmanabhan, S., Singh, S., Rutledge, A. and Triggle, D.J. (2000) Permanently Charged Chiral 1,4-Dihydropyridines: Molecular Probes of L-Type Calcium Channels. Synthesis and Pharmacological Characterization of Methyl ( $\omega$-Trimethylalkylammonium) 1,4-Dihydro-2,6-Dimethyl-4-(3-Nitrophenyl)-3,5-Pyridinedicarboxylate Iodide. Calcium Channel Antagonists. Journal of Medicinal Chemistry, 43, 2906-2914. http://dx.doi.org/10.1021/jm0000281

[9] Zhou, X., Zhang, L., Tseng, E., Scott-Ramsay, E., Schentag, J.J., Coburn, R.A. and Morris, M.E. (2005) New 4-Aryl1,4-Dihydropyridines and 4-Arylpyridines as p-Glycoprotein Inhibitors. Drug Metabolism and Disposition, 33, 321328. http://dx.doi.org/10.1124/dmd.104.002089

[10] Karade, N.N., Gampawar, S.V., Kondre, J.M. and Shinde, S.V. (2008) An Efficient Combination of Dess-Martin Periodinane with Molecular Iodine and $\mathrm{KBr}$ for the Facile Oxidative Aromatization of Hantzsch 1,4-Dihydropyridines. Arkivoc, xii, 9-16. http://dx.doi.org/10.3998/ark.5550190.0009.c02

[11] Cheng, D.P. and Chen, Z.C. (2002) Hypervalent Iodine in Synthesis. 76. An Efficient Oxidation of 1,4-Dihydropyridines to Pyridines Using Iodobenzene Diacetate. Synthetic Communications, 32, 793-798. http://dx.doi.org/10.1081/SCC-120002521

[12] Kumar, P. (2009) Solid State Oxidative Aromatization of Hantzsch 1,4-Dihydropyridines to Pyridines Using Iodobenzene Diacetate or Hydroxy(tosyloxy)iodobenzene. Chinese Journal of Chemistry, 27, 1487-1491. http://dx.doi.org/10.1002/cjoc.200990250

[13] Kumar, P. (2010) A Novel, Facile, Simple and Convenient Oxidative Aromatization of Hantzsch 1,4-Dihydropyridines to Pyridines Using Polymeric Iodosobenzene with KBr. Journal of Heterocyclic Chemistry, 47, 1429-1433.

[14] Lee, K.H. and Ko, K.Y. (2002) Aromatization of Hantzsch 1,4-Dihydropyridines with [Hydroxy(tosyloxy)iodo] Benzene. Bulletin of the Korean Chemical Society, 23, 1505-1506.

[15] Lee, J.W. and Ko, K.Y. (2004) Aromatization of Hantzsch 1,4-Dihydropyridines with a Polymer-Supported Hypervalent Iodine Reagent. Bulletin of the Korean Chemical Society, 25, 19-20.

[16] Varma, R.S. and Kumar, D. (1999) Solid State Oxidation of 1,4-Dihydropyridines to Pyridines Using Phenyliodine(III)Bis(trifluoroacetate) or Elemental Sulfur. Journal of the Chemical Society, Perkin Transactions 1, 24, 1755-1757.

[17] Varvoglis, A. (1997) Chemical Transformations Induced by Hypervalent Iodine Reagents. Tetrahedron, 53, 1179-1255. http://dx.doi.org/10.1016/S0040-4020(96)00970-2

[18] Wirth, T., Chiai, M., Zhdankin, V.V., Koser, G.F., Tohma, H. and Kita, Y. (2003) Topics in Current Chemistry. Vol. 224, Springer, Berlin.

[19] Zhdankin, V.V. and Stang, P.J. (2008) Chemistry of Polyvalent Iodine. Chemical Reviews, 108, 5299-5358. http://dx.doi.org/10.1021/cr800332c

[20] Böcker, R.H. and Guengerich, F.P. (1986) Oxidation of 4-Aryl- and 4-Alkyl-Substituted 2,6-Dimethyl-3,5-bis(alkoxy- 
carbonyl)-1,4-dihydropyridines by Human Liver Microsomes and Immunochemical Evidence for the Involvement of a Form of Cytochrome P-450. Journal of Chemical Education, 29, 1596-1603.

http://dx.doi.org/10.1021/jm00159a007

[21] Rajput, A.P. and Rajput, S.S. (2011) A Novel Method for the Synthesis of Formyl Pyrazoles Using Vilsmeier-Haack Reaction. International Journal of Pharmacy and Pharmaceutical Sciences, 3, 346-351.

[22] Kumar, P., Kumar, A. and Hussain, K. (2012) Iodobenzene Diacetate (IBD) Catalyzed an Quick Oxidative Aromatization of Hantzsch-1,4-dihydropyridines to Pyridines under Ultrasonic Irradiation. Ultrasonics Sonochemistry, 19, 729735. http://dx.doi.org/10.1016/j.ultsonch.2011.12.021 
Scientific Research Publishing (SCIRP) is one of the largest Open Access journal publishers. It is currently publishing more than 200 open access, online, peer-reviewed journals covering a wide range of academic disciplines. SCIRP serves the worldwide academic communities and contributes to the progress and application of science with its publication.

Other selected journals from SCIRP are listed as below. Submit your manuscript to us via either submit@scirp.org or Online Submission Portal.
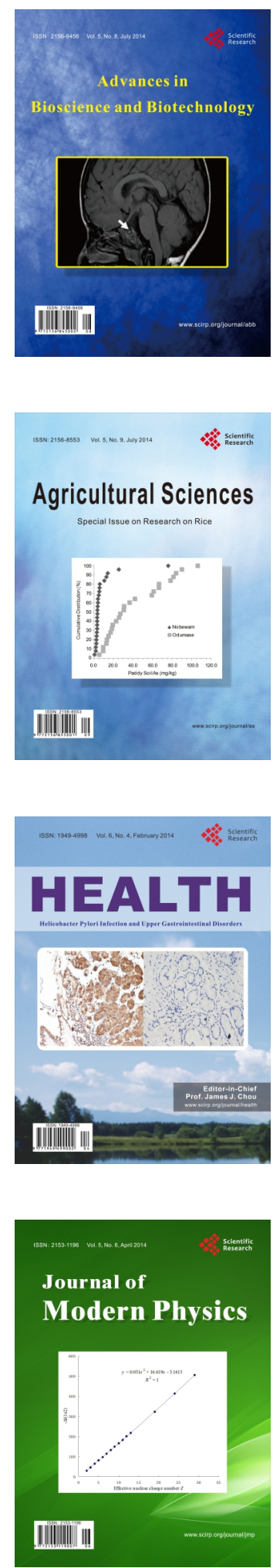
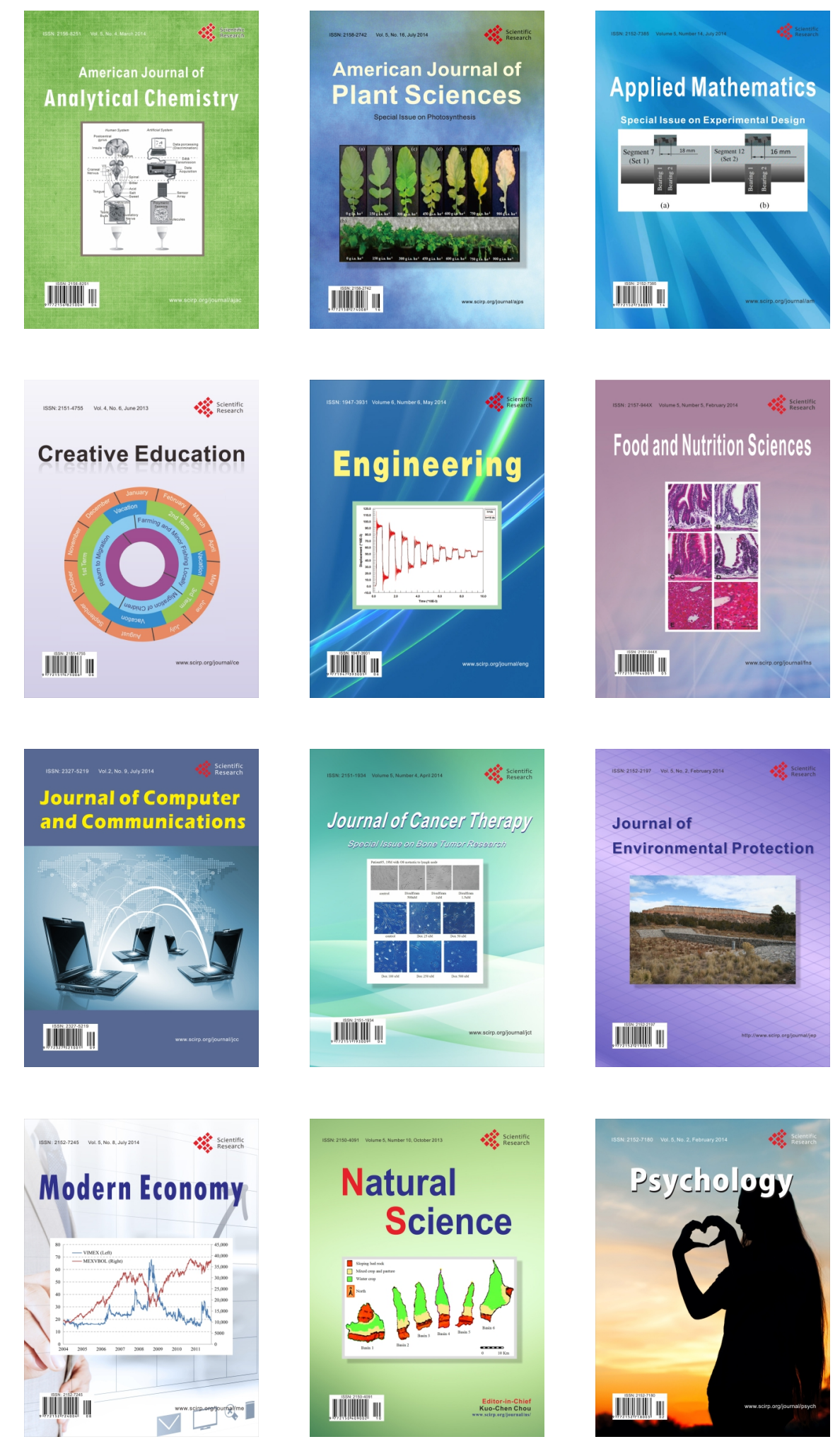